\title{
El foro invertido en la movilización del conocimiento: alcances e implicaciones
}

\author{
The flipped forum in knowledge mobilization: Scope and implications
}

\author{
Elieth Diez ${ }^{a}$ ORCID: 0000-0003-3606-6215 \\ Zuly Del Fiaco ${ }^{b}$ ORCID: 0000-0001-8043-544X \\ Alberto Castellano ${ }^{c}$ ORCID: 0000-0001-8244-1894 \\ Luis Ordońez ${ }^{\mathrm{d}}$ ORCID: 0000-0001-9750-0674
}

Recibido: 26/10/2019・ Aprobado: 31/01/2020

Cómo citar: Diez, E., Del Fiaco, Z., Castellano, A., \& Ordoñez, L. (2020). El foro invertido en la movilización del conocimiento: alcances e implicaciones. Ciencia y Educación, 4(1), 7-20. Doi: https://doi.org/10.22206/cyed.2020.v4i1.pp7-20

\section{Resumen}

Este artículo sintetiza los alcances y las implicaciones del foro invertido Movilización del conocimiento entre Universidad y Sociedad, desarrollado por la Fundación InterConectados (Venezuela). Este foro, inspirado en la clase invertida, es una estrategia apoyada en las TIC, concebido como un espacio para gestionar y movilizar conocimiento e información, producto de tareas investigativas donde se promueve la colaboración y la interacción, con la pretensión de configurar nuevas ecologías de aprendizaje. Se realizó una revisión documental sobre gestión y movilización del conocimiento, enfoque invertido en educación, TIC y foros académicos. El enfoque fue vivencialista-experiencialista, mediante el método fenomenológico. A partir de un muestreo intencional, se recolectó la información a través de dos técnicas, como parte del proceso de triangulación: la entrevista semi-estructurada con 2 informantes clave que participaron en varios foros y un cuestionario de preguntas abiertas aplicado a 14 informantes clave. Tras un análisis cualitativo, los hallazgos mostraron mayor

\begin{abstract}
This article summarizes the scope and implications of the flipped forum Mobilization of knowledge between University and Society, developed by the Fundación InterConectados (Venezuela). The forum based in a flipped classroom is a strategy supported by ICT, conceived as an space to manage and mobilize knowledge and information, as the result of research tasks, and where collaboration and interaction are promoted, with the aim of setting up new learning ecologies. A brief documentary review was made on knowledge management and mobilization, flipped approach in education, ICT and academic forums. The review was conceived from an experientialist approach, using the phenomenological method. Based on intentional sampling, the information was collected, and two techniques were used as a part of the triangulation process. At first, the semi-structured interview with 2 participants of different issues of the forums, while the second time a questionnaire was applied to 14 voluntary participants. After a qualitative analysis, the findings showed a greater
\end{abstract}

\footnotetext{
a Fundación InterConectados, Venezuela. Correo-e: eliethdiez@gmail.com

b Fundación InterConectados, Venezuela. Correo-e: zdelfiacco@gmail.com

c Fundación InterConectados, Venezuela. Correo-e: albertocastellanog@gmail.com

d Fundación InterConectados, Venezuela. Correo-e: lordonezv@gmail.com
} 
alcance e implicación del foro invertido en la dimensión social, pero también en lo educativo y organizacional; esta última recibió una valoración positiva, al dejar en claro la necesidad de mejorar aspectos relacionados con la estructura y el funcionamiento para la optimización del foro.

Palabras clave: foro invertido; movilización de conocimiento; ecologías de aprendizaje; TIC; aprendizaje no formal.

\section{Introducción}

La Fundación InterConectados ha venido realizando el foro Movilización del conocimiento entre Universidad y Sociedad, en el marco de la Convención Anual de la Asociación Venezolana de la Ciencia (AsoVAC), con el apoyo de la presidencia de esta organización. Este foro, a lo largo del tiempo, ha ido transformándose con la idea de perfeccionar un modelo de herramienta tecnológica que se consolide como espacio idóneo para la comunicación de la información y el conocimiento útil para la sociedad, es decir, un contexto y una estrategia que contribuya a expandir el conocimiento y a enriquecer las ecologías emergentes del aprendizaje, las cuales dan cuenta de los cambios dados en su concepción, "dónde, cuándo, y cómo aprendemos, con quién y de quién aprendemos, y también qué aprendemos y para qué" (Coll, 2013, p. 3). De manera que el foro se percibe, en este momento, como proveedor de un contexto para la gestión-movilización del conocimiento y del aprendizaje que reúne el "carácter social, comunitario, participativo e interactivo presente en las nuevas ecologías del aprendizaje" (Fernández \& Martínez, 2018, p. 19).

El foro requiere de una evaluación permanente $y$, en esta ocasión, se pretendió revelar sus alcances e implicaciones desde la perspectiva de los participantes; así, se comienza con una breve revisión documental sobre la gestión-movilización del conocimiento y el enfoque invertido en educación, destacando los foros académicos y las TIC, en especial, el foro invertido; aunque este no aparece en la literatura con tal denominación, ha sido definido en esta investigación como scope and implication of the forum invested in the social dimension, but also in the educational and organizational ones. The latter, although it received a positive assessment, the need to improve aspects related with the structure and functioning for the optimization of the forum.

Keywords: Flipped forum; mobilization of knowledge; ecologies of learning; ICT; non-formal learning.

un evento académico virtual donde los interesados pueden participar mediante dos fases -asincrónica y sincrónica- para discutir sobre una temática. Tal revisión sirve como soporte para facilitar la comprensión de los hallazgos, la reflexión y el reconocimiento de sus potencialidades como herramienta tecnológica apropiada para realizar intervenciones más efectivas en el futuro, además de permitir difundir esta modalidad de foro invertido para que tenga proyección en ambientes organizacionales con requerimientos de aprendizaje digital en procesos relacionados con la gestión y la movilización de conocimiento (Dougherty \& Dunne, 2011; Adell \& Castańeda, 2012).

\subsection{Gestión-movilización de conocimiento y TIC}

La importancia del conocimiento ha sido un tema tratado en distintas investigaciones en las últimas décadas (Hayek, 1945; Grant, 1996; Drucker, 2000); concebido como un recurso económico altamente valorado (Rodríguez, García, Pérez \& Castillo; 2009) que necesita ser gestionado. De acuerdo con Nonaka \& Takeuchi (1995), la gestión del conocimiento se define como la capacidad de "crear conocimiento nuevo, diseminarse en la organización e incorporarlo en productos, servicios y sistemas" (p. 3); o sea, desarrollar la capacidad de articular los recursos que se disponen para su producción, organización, procesamiento y difusión (Grant, 1996b; García, 2010).

Luego, surgió el concepto $\mathrm{K}+$ como un término que agrupa una serie de funciones y procesos, vinculando el conocimiento con la práctica y las políticas públicas, mejorando las formas en que este es compartido y aplicado. Según Shaxson, Bielak, Ahmed, 
Brien, Conant, Fisher \& Phipps (2012), en el K+ se destaca la movilización de conocimiento como proceso y la gestión de las organizaciones intermediarias como mediadores en las relaciones recíprocas que existen entre los productores y los usuarios del conocimiento (universidades y comunidad). De acuerdo con Anderson \& Mclachlan (2016), existen tres estrategias para favorecer la movilización de conocimiento entre el mundo académico y no académico: (a) estratificar el conocimiento; (b) construir espacios de diálogo; (c) usar diferentes medios de comunicación.

Las estrategias ya referidas son facilitadas aprovechando el rol de las TIC, en tanto son herramientas que permiten a las personas involucradas comunicarse e interactuar en diferentes niveles del sistema social (Fisher, 2012). De esta manera, cada organización que aprende necesita conformar un modelo de gestión que constituya una ecología de aprendizaje organizacional propia con un doble propósito: la inserción en el sistema de conocimiento mayor y, a su vez, la creación de ecologías de aprendizaje individuales, al tiempo que se requiere movilizar conocimiento entre diferentes organizaciones. Así que la gestión-movilización del conocimiento, enmarcado en las ecologías del aprendizaje, da protagonismo a las TIC porque ellas proporcionan, cada vez más, espacios originales que incrementan las oportunidades, los recursos y las estrategias para movilizar el conocimiento, así como para que se generen aprendizajes sustentados en entornos colaborativos con procesos formales, informales y no formales (Williams, Karousou \& Mackness, 2011; Sangrà \& Wheeler, 2013).

\subsection{Gestión-movilización de conocimiento y el enfoque invertido en foros académicos}

La gestión del conocimiento en las organizaciones de cualquier naturaleza requiere de las TIC como soporte tecnológico para distintas actividades y diferentes procesos, incluidos los de aprendizaje, al ser consideradas como medios para gestionar el conocimiento (Bañuelos \& Barron, 2005). Igualmente, en procesos de difusión y movilización de conocimiento las TIC constituyen un medio ideal que facilita la comunicación, la interacción, el trabajo colaborativo, el diálogo, la transferencia o la construcción de nuevos aprendizajes, lo que da oportunidad a que eventos de distinta índole aprovechen las herramientas tecnológicas para ser realizados de manera virtual.

De acuerdo con Quirós (2009), las TIC aplicadas en la educación han permitido facilitar procesos de creación de contenido, escenarios de formación, entornos de aprendizaje y el desarrollo de otros recursos digitales innovadores para el trabajo colaborativo.

Adicionalmente, el enfoque invertido en educación ha ganado espacios de investigación desde 2012 (Karabulut-Ilgu, Jaramillo \& Jahren, 2018). Tiene distintas aplicaciones en la práctica educativa, siendo la más común la clase invertida; pero, diversos estudios dan cuenta de su aplicación en cursos, talleres, seminarios que se celebran con distintos fines, entre ellos los relativos a gestión y movilización de conocimiento (Milman, 2012; Herreid \& Schiller, 2013; Abeysekera \& Dawson, 2015; Zainuddin \& Halili, 2016). La clase invertida es una estrategia de aprendizaje activo, autónomo y reflexivo del participante, donde este se convierte en el centro de la clase, teniendo un rol más autónomo y con un enfoque de trabajo y aprendizaje práctico y aplicable fuera del aula (Baker, 2000; Herreid \& Schiller, 2013; Butt, 2014; O’Flaherty \& Phillips, 2015).

La autonomía y el trabajo previo y reflexivo del participante son elementos comunes en la clase invertida y en lo que se ha denominado foro invertido, un evento académico virtual donde los interesados pueden participar mediante dos fases -asincrónica y sincrónica- para discutir sobre una temática; mientras que la diferencia entre ambos es que la clase invertida puede valerse de herramientas TIC para facilitar el conocimiento, pero el foro invertido utiliza las TIC a lo largo de todo el proceso. Esta innovación del foro invertido lo convierte en una estrategia que se vale de las herramientas tecnológicas para favorecer la interacción a distancia y permitir la discusión entre diferentes personas sobre un tema particular. Como consecuencia, el foro invertido se convierte en lo que distintos autores han denominado eventos virtuales 
o foros electrónicos, es decir, un espacio de gestión de conocimiento utilizado entre pares para compartir los resultados de sus investigaciones (Brito, 2004; Wu \& Hiltz, 2004; Buil, Hernández, Sesé \& Urquinzi, 2012; Crespo, 2015).

\subsection{Foro invertido de InterConectados}

La Fundación InterConectados y su bitácora (blog) nació en 2011 (Ordóñez Vela, 2016) para contribuir, con el apoyo de las TIC, a la generación, recopilación y movilización de conocimientos entre la universidad y la sociedad latinoamericana. Con el uso de Internet y la web 2.0 para facilitar la información y la comunicación, la fundación ha logrado: (a) el dictado de cursos abiertos masivos en línea (CAMEL, del acrónimo en inglés MOOC); (b) la creación de oportunidades para la reflexión sobre los problemas culturales que dificultan la colaboración en nuestro medio (más de 250 entradas en la bitácora; (c) la interacción con docentes, investigadores y comunidades mediante boletines; y (d) la organización de foros sobre movilización de conocimiento entre universidad y sociedad, conformando así un sistema o ecología de aprendizaje sobre el tema (ver Figura 1). El propósito general de InterConectados, con base en las tecnologías disponibles, es el desarrollo de capital humano y social que, junto con las leyes, las normas y los reglamentos que se puedan desarrollar, faciliten la generación e integración de conocimiento útil para la toma de decisiones, e insertar este conocimiento en los procesos de definición de políticas públicas para así contribuir a la solución de los problemas que aquejan a la sociedad.

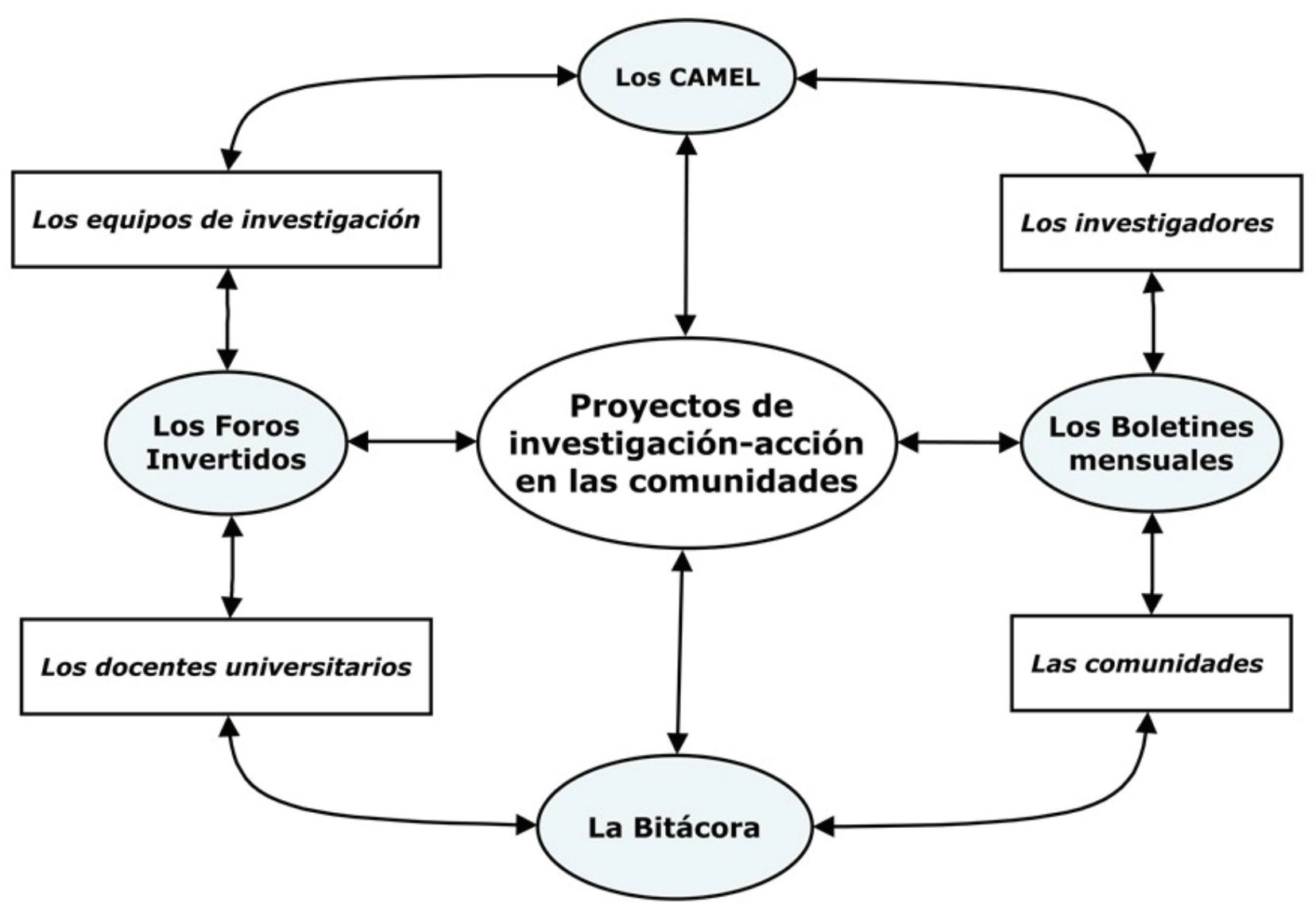

Figura 1. La Fundación Interconectados como sistema interactivo

Fuente: elaboración propia. 
El foro académico tradicional o presencial fue utilizado originalmente por la Fundación InterConectados en el año 2013, en el seno de la Convención Anual de la Asociación Venezolana para el Avance de la Ciencia (AsoVAC). Se pretendía constituir un espacio de comunicación e intercambio para la discusión de experiencias relativas a la movilización de conocimiento entre universidad y sociedad, en especial, los productos de las investigaciones desarrolladas por los docentes, en su función de investigación y demás indagaciones asociadas con el quehacer universitario, como el servicio comunitario y cualquier otra forma de intercambio con la sociedad de la que disponga la universidad (Díez \& Ordoñez; 2018). Entre el foro II y III se fueron introduciendo las presentaciones en video, pregrabadas en YouTube, pero presentadas en reunión virtual de los participantes de forma sincrónica. Para el IV foro, en el año 2016, InterConectados decide hacer un cambio para utilizar una metodología de foro invertido, inspirados en la clase invertida (flipped classroom), estrategia que tuvo un impacto positivo en el desarrollo de la actividad y dio lugar al evento virtual que recibe el nombre de foro invertido.

El foro invertido de InterConectados se constituye así en una estrategia válida para capturar, preservar y difundir el conocimiento, representando un valor agregado en este tipo de actividades en línea, lo que a su vez se traduce en procesos de gestión del conocimiento donde la información (transformada en conocimiento) se consigue en forma precisa para la persona apropiada, en el momento oportuno y, además, proporciona herramientas para el ejercicio del análisis y la capacidad de respuesta ante las ideas que allí se debaten. Estudiar sus alcances e implicaciones en el formato actual y explorar nuevas formas organizativas que optimicen sus logros justifica su estudio.

\section{Material y método}

Para denotar las percepciones de los participantes acerca de los alcances del foro como herramienta TIC y sus implicaciones, así como para puntualizar los aspectos organizacionales susceptibles de cambio, se privilegió el enfoque epistemológico vivencialista-experiencialista, soportado por la subjetividad (Padrón, 2016); y para concretar las acciones procedimentales y operativas se asumió el método fenomenológico, en su tendencia de la fenomenología hermenéutica, que se centra en el estudio de las realidades experimentadas por los sujetos, tal como son vividas. Se recabó la información, tomando en cuenta dos momentos de realización del foro (2017 y 2018); en el primer momento, se usó la técnica de la entrevista y los informantes fueron seleccionados de manera intencional (Hernández, Fernández \& Baptista, 2014), según ciertos criterios de pertinencia y adecuación planteados por Tójar (2006), por lo que solo dos (2) de tres (3) personas que cumplían con los criterios de haber participado en por lo menos dos (2) ediciones del foro, en condición de ponentes, aceptaron hacer la entrevista.

Para el segundo momento, se consideró la técnica del cuestionario abierto en línea, el cual fue respondido por 14 personas usando los mismos criterios y las mismas condiciones ya mencionados. Es importante destacar que el número de informantes no es lo relevante en el muestreo cualitativo, "lo relevante es no dejar a un lado información que pudiera ser esencial para la investigación que se está desarrollando" (Tójar, 2006 p. 187) y la cantidad depende de la saturación de información, es decir, que cuando ya no se encuentra nada nuevo, no es preciso incorporar más informantes. El análisis cualitativo se hizo con el proceso de categorización y estructuración.

Las preguntas de la entrevista se asociaron con unos elementos predeterminados para que los participantes pudieran manifestar sus experiencias en el foro y los significados atribuidos, los cuales se identificaron como: (a) tema del foro; (b) metodología del foro; (c) relevancia del foro; (d) resultados del foro. Por cada elemento se hicieron las preguntas pertinentes según la dinámica de la entrevista y los aportes de las personas entrevistadas. Para la segunda recolección de información mediante el cuestionario en línea se hicieron tres (3) preguntas, construidas de forma abierta, una (1) relacionada con la fase de organización y dos (2) con la fase de ejecución. La intención de dichas preguntas pretendió recoger información sobre las mejoras que pudieran darse en las distintas fases de la gestión del foro, pensando en la creación de una metodología de trabajo idónea para este. 


\section{Resultados}

\subsection{Alcances e implicaciones del foro invertido}

La información requerida a los informantes fue transcrita en su totalidad y sometida al proceso de categorización y estructuración correspondiente, representado por el reconocimiento de las unidades de significado (respuestas textuales dadas por los entrevistados), las categorías individuales y las generales o emergentes, además de la síntesis interpretativa. Para este artículo se obvió el paso de las unidades de significado a categorías individuales, así se presentan directamente las categorías individuales y las generales o emergentes, de forma gráfica, mientras que la síntesis interpretativa se da explícitamente en el texto.

De esta manera, en la figura 2 se muestran las categorizaciones de lo individual a lo general o emergente, así se comienza con: (a) el tema del foro, que congrega dos (2) categorías generales: primero, la oportunidad de comunicar e intercambiar experiencias e ideas, la cual alude a la aceptación del foro y su temática como promotor de la comunicación, en múltiples direcciones, sobre las producciones académicas individuales y grupales. La otra categoría fue la de organización y efectos del foro, que refiere la presencia de estrategias y pautas de orden organizativo y de gestión mediadas por las TIC, lo que representa una apreciación favorable hacia el foro desde el punto de vista organizacional y temático.
Luego, se continuó con (b) la metodología del foro (figura 2), así se exhiben dos categorías generales sintetizadas en lo que se denominó aspectos actitudinales ante las TIC y el trabajo en comunidades, que tiene que ver con el conjunto de actitudes o posturas asumidas ante el uso de tecnologías, su papel en procesos de aprendizaje y el trabajo con comunidades. Mientras la segunda fue denominada alcances y limitaciones de la metodología del foro, haciendo referencia a los beneficios y las posibilidades derivados de la metodología empleada y las limitaciones que obstaculizan su implementación.

Sobre la tercera temática, (c) la relevancia del foro, los resultados en la figura 2 se resumen en dos (2) categorías generales; primero, compartir conocimiento, entendida como una oportunidad de participar en un evento virtual para difundir conocimientos y prácticas con otros interesados en el tema; mientras que la segunda categoría general denominada trabajo colaborativo se refiere a los significados, intereses y valores atribuidos a realizar investigaciones junto con otros colegas y las implicaciones que tiene el proceso de colaborar. También se visualizan las categorías referidas a la última temática, (d) resultados del foro, con la identificación de dos (2) categorías generales relativas; primero, motivación e interés en trabajo colaborativo y luego la comprensión del valor de las TIC en el trabajo. Estas categorías traen a colación el tema del trabajo colaborativo como un punto central imbricado por la motivación, los alcances y la valoración de las TIC, lo que hace inferir unos resultados positivos del foro entre los participantes.

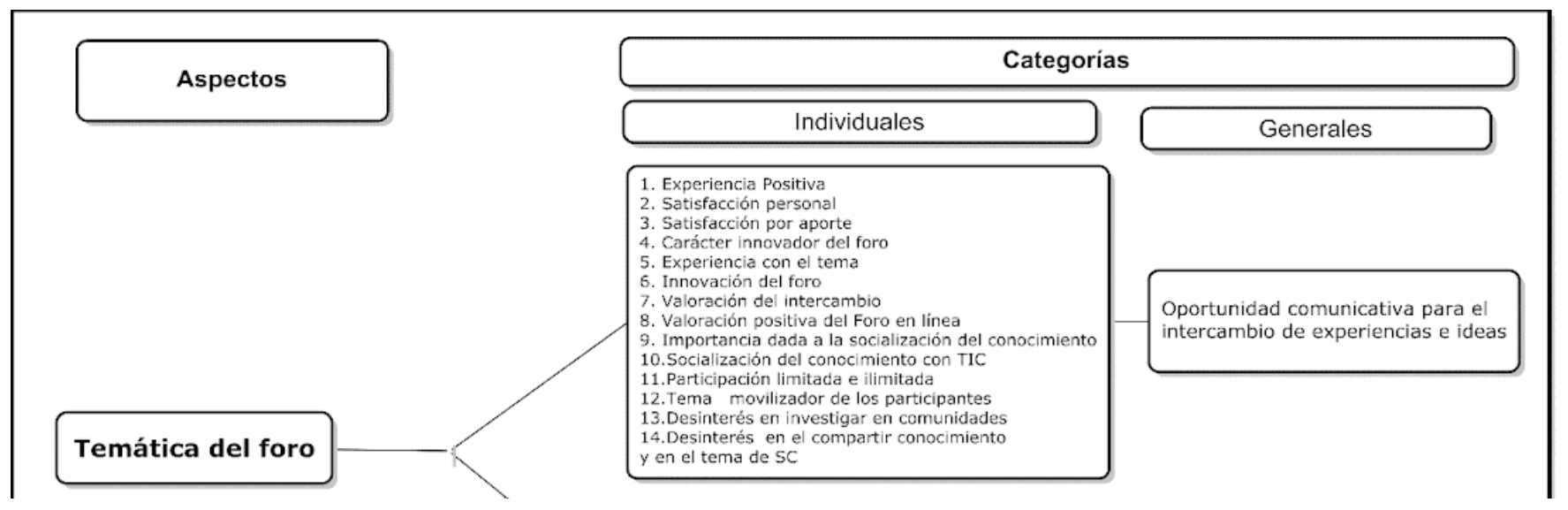




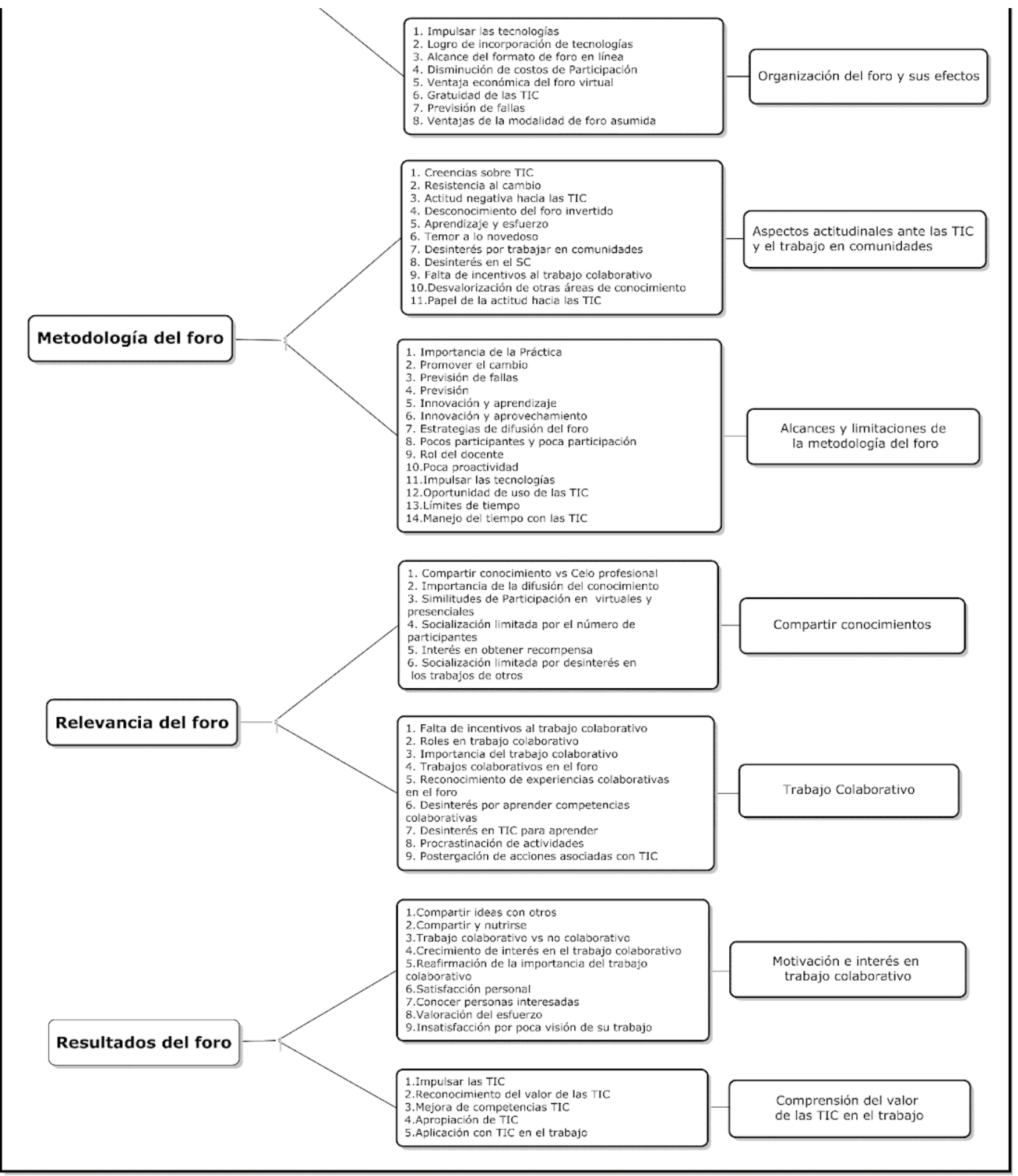

Figura 2. Categorización de alcances e implicaciones del foro invertido

Fuente: elaboración propia. 
Las categorizaciones dan lugar a la estructuración general del foro invertido, como puede observarse en la figura 3, a partir de las categorías generales o emergentes que permiten considerar unos alcances y sus implicaciones en las dimensiones de lo social, educativo y organizacional. En lo social, encajan cuatro (4) categorías emergentes: oportunidad comunicativa para el intercambio de ideas y experiencias, compartir conocimientos, trabajo colaborativo y motivación e interés en trabajo colaborativo; estas categorías permiten reafirmar que el foro invertido es un espacio para la comunicación, la interacción y el diálogo, lo que hace fluir el conocimiento, de forma explícita y tácita, un contexto ideal para la conformación de ecologías de aprendizaje.

En lo educativo, el foro tiene su alcance, representado en dos categorías emergentes, una referida a los aspectos actitudinales ante las TIC y el trabajo en comunidades; la otra, comprensión del valor de las TIC en el trabajo. La primera remite a la necesidad de formarse en las TIC para el cambio actitudinal ante estas al reconocer el papel que juegan, mientras que la segunda da una valoración positiva para el uso de las TIC en la docencia, la investigación y la extensión. La categoría organización del foro y sus efectos, sumada a los alcances y las limitaciones de su metodología dan lugar al alcance organizacional, al reconocer aspectos de su gestión y economías del uso de tiempo, lugar y costos, además de las posibilidades y los beneficios como herramienta virtual.

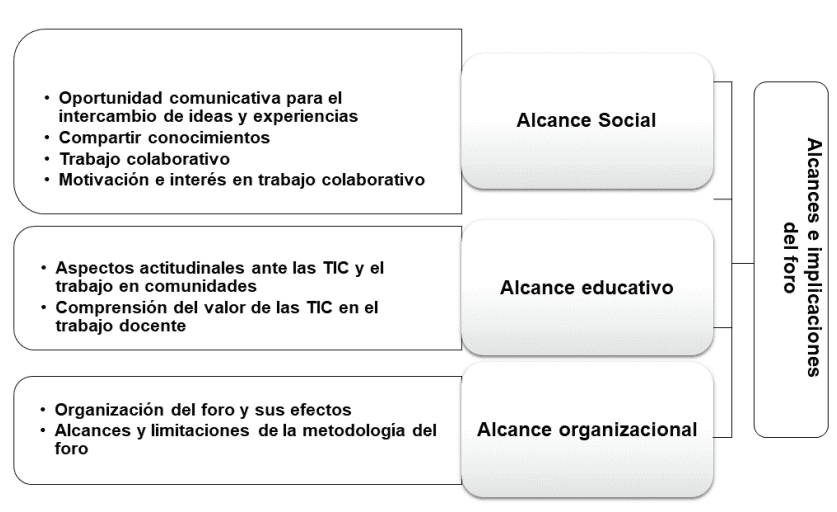

Figura 3. Estructura de alcances e implicaciones del foro

Fuente: elaboración propia.

\subsection{Alcances organizacionales del foro invertido susceptibles de cambio}

La información recogida en un segundo momento, orientada a la dimensión organizacional del foro, se presenta en la figura 4, con lo correspondiente a la fase de planificación, donde pueden distinguirse directamente la concreción de cinco (5) categorías emergentes resultantes de la agrupación de categorías individuales reconocidas, que son: (a) necesidad de dar una información más descriptiva y precisa sobre el foro como herramienta, además de lo concerniente a su implementación; es decir, mejorar su descripción, su metodología, las condiciones, las instrucciones, la naturaleza y las temáticas de los trabajos, la población a la que se dirige, los ponentes. Prosigue la categoría (b) tiempo, como una variable además de ser condición necesaria, desde la planificación hasta la ejecución y evaluación del evento, al considerar elementos como informar y promover con anticipación el evento, los lapsos para la inscripción de ponencias, los requerimientos, la entrega de las ponencias, las fechas para las fases asincrónica y sincrónica, entre otras.

Una tercera categoría (c) implica una campaña promocional del evento que resulte atractiva y efectiva, la cual debe sostenerse en el tiempo para mantener el foro como una actividad de relevancia y de interés para ciertas comunidades; al respecto, los informantes comentaron "Lo único que hubiera mejorado un poco más de promoción, quizás haciendo material y dándolo a los ponentes para circular en redes sociales, al estilo afiche o volante". (Informante 5)

Luego, se identificó la categoría correspondiente a la (d) definición de líneas o áreas temáticas, para congregar distintos trabajos realizados o que estén realizando los ponentes que se identifiquen con la razón principal del foro. La quinta categoría se refiere a la (e) muestra de trabajos anteriores, lo que puede servir para incentivar y valorar el foro como un escenario propicio para presentar y divulgar trabajos, cuyos aportes pueden ser útiles o interesantes para las comunidades a quienes este se dirija.

También, en la figura 4 se presentan las categorías asociadas con la fase asincrónica, reflejando la percepción de los investigadores sobre las expresiones 
negativas de los informantes ante varias acciones y que se pudieron resumir en tres (3) categorías emergentes. Primero emergió (a) la selección de un espacio apropiado para lo asincrónico; en este punto, el informante 2 comentó: "si utilizara una plataforma seria, confiable y organizada que permita hacer todo en el mismo lugar..."; es decir que en la fase asincrónica el utilizar varios espacios para la misma actividad trae confusión, la mayoría de los informantes se identificaban con YouTube como espacio ideal para tal fin. Entonces, el contar con un solo espacio permite que no se dispersen las opiniones de los participantes y facilita el trabajo de interacción y recolección de las intervenciones para su presentación posterior.

La siguiente categoría fue identificada como (b) la promoción y dinamización de la actividad, que se convierte en una necesidad para incrementar la audiencia; por ejemplo, el informante 6 mencionó que podían mejorarse "incentivando a los profesores a participar más en cuanto a los comentarios realizados"; es así como la dinamización se centra en el incremento de la participación de la audiencia, a través de sus opiniones y comentarios acerca de las ponencias que se presenten. Finalmente, se identificó (c) la previsión de la optimización del tiempo para que los participantes vean las ponencias y las comenten.
Asimismo, la figura 4 refleja las categorizaciones asociadas con la fase sincrónica, para la cual se enumeraron tres (3) categorías emergentes: la primera fue las condiciones de lugar, plataforma única y previsión de la conexión, es decir, se necesita un lugar que facilite la organización previa del evento sincrónico, el uso de una sola plataforma que funcione adecuadamente y debe tenerse la previsión de una conexión que satisfaga los requerimientos del evento. Otra categoría identificada fue la audiencia, o sea, la población objeto del evento, pues se predica que es movilización de conocimiento de la universidad para la sociedad; al entender del informante 3 "...se trataba de un evento de la universidad para la universidad...", se percibe entonces que la audiencia sigue siendo la universidad. Además, se identificó una tercera categoría denominada reforzar la fase asincrónica, apuntando al logro suficiente de audiencia, participación e interacción para que la fase sincrónica pueda contar con suficientes elementos para los comentarios finales por ponencia, optando además con las posibilidades de una fase presencial que pueda ser organizada por grupos de interés. En este particular, el informante 8 apuntó: "Como aporte si vuelvo a participar, creo que organice una jornada presencial, en donde se proyecten los videos previamente y el vivo la fase sincrónica”.

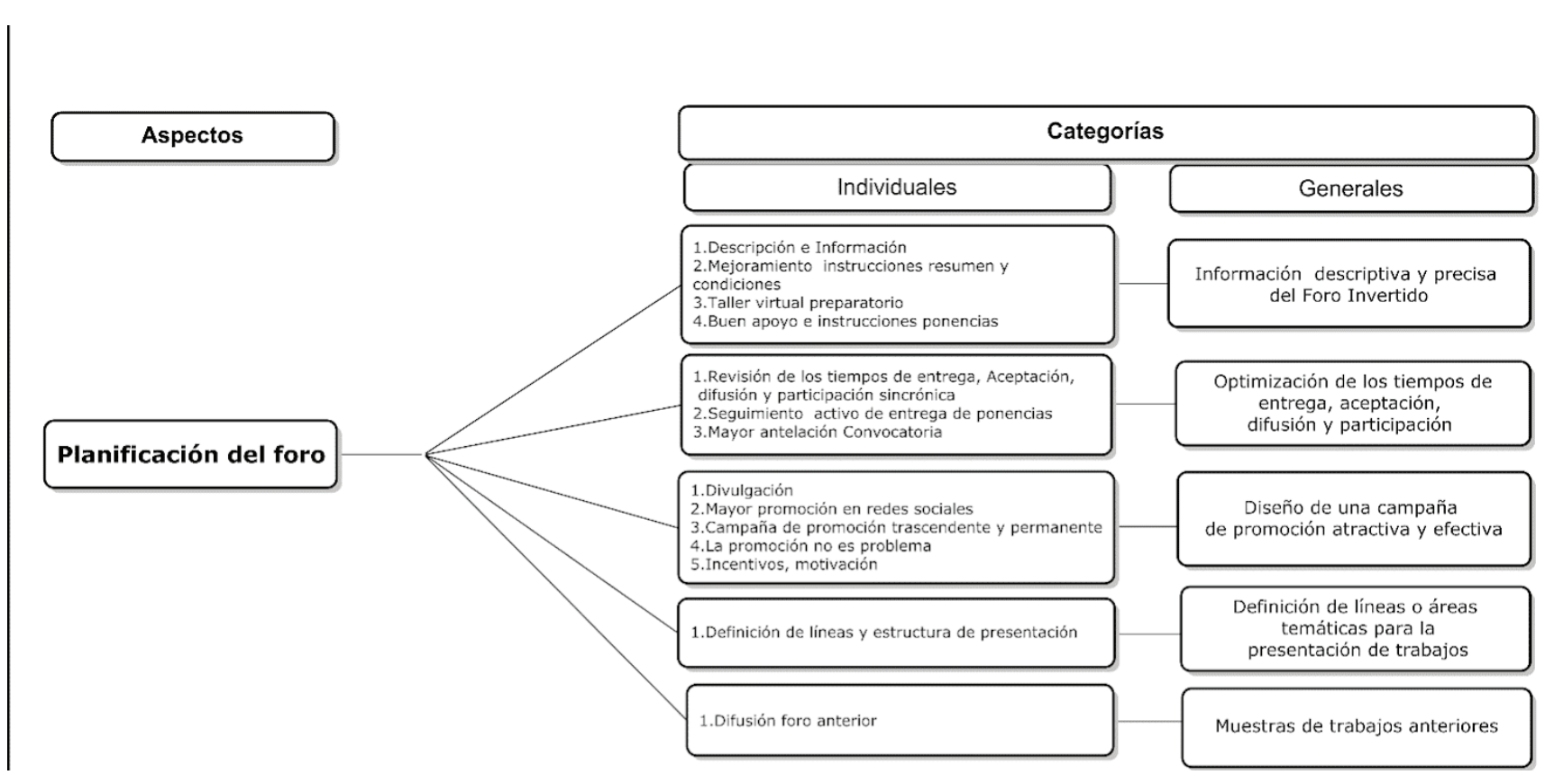




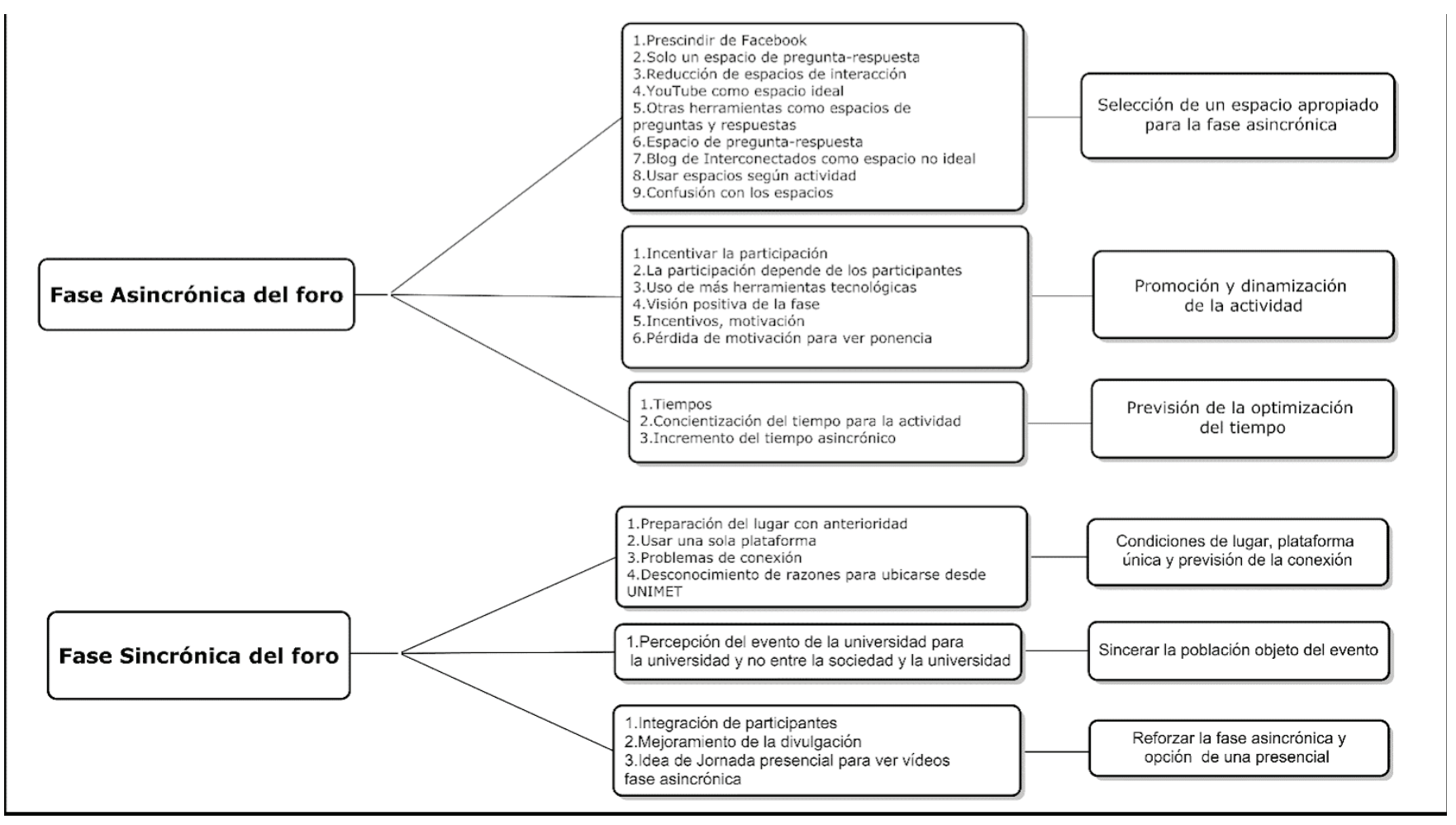

Figura 4. Categorización de alcances organizacionales para el mejoramiento del foro invertido.

Fuente: elaboración propia.

La estructuración de los alcances organizacionales del mejoramiento del foro se muestra en la figura 5, donde se puede notar que las categorías generales o emergentes se sintetizaron en dos grandes categorías que permiten concretar las dimensiones organizacionales a las cuales se debe prestar atención, así puede decirse que las mejoras del foro se centrarán en las dimensiones de: (a) información y comunicación y (b) tiempo, espacio y conexión, con la idea de que el foro se consolide como espacio para la gestión y movilización del conocimiento, además del aprendizaje. Tales cuestiones deben ser tomadas en cuenta para la preparación y organización de los futuros foros que ofrezca InterConectados, lo que remite a considerar su estructura y funcionamiento, a partir de los hallazgos de esta indagación.

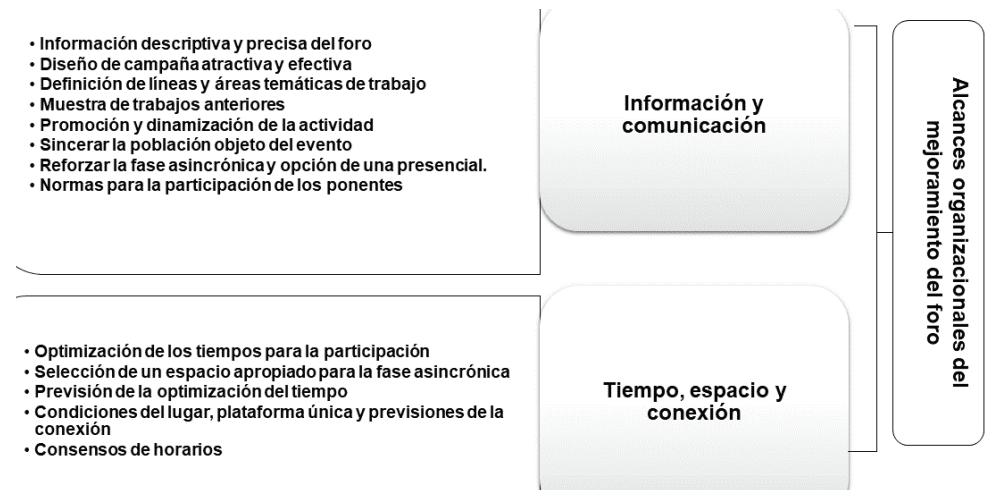

Figura 5. Estructuración de alcances organizacionales del mejoramiento del foro

Fuente: elaboración propia. 


\section{Discusión y conclusiones}

Dado el carácter inacabado de las investigaciones desarrolladas con el método fenomenológico, se presentan algunas conclusiones transitorias a efectos de discusión en lo que concierne a las interrogantes que dieron lugar al estudio: ¡cómo percibieron los informantes el foro invertido como herramienta TIC y sus implicaciones? y ¿cuáles aspectos relativos a la organización del foro pueden ser mejorados u optimizados?

En cuanto a las percepciones de los alcances y las implicaciones que sobre el foro invertido manifestaron los informantes se destaca su alcance y efecto en lo social como herramienta tecnológica, ya que a través de él se producen interacciones, intercambios y dinámicas alrededor de temas comunes y de interés para los participantes, vistos desde diferentes ópticas que facilitan el intercambio, la colaboración y la construcción de conocimientos de forma expansiva, además de las posibilidades de impactar a un mayor número de personas por las facilidades que da la virtualidad (Martínez, Esquivel \& Martínez, 2014; Herreid \& Schiller, 2013). El foro invertido cumple un doble objetivo: gestionar conocimiento a lo interno de la organización y movilizar conocimiento entre los participantes, configurándose como un elemento que favorece las ecologías de aprendizaje.

Se suma el alcance y la implicación educativa de carácter no formal, a través del foro invertido, al remitir a una práctica con TIC y con la movilización de conocimientos, lo cual conduce a promover cambios en el saber, el ser y el hacer; estos cambios son aprendizajes logrados de manera no intencional, es aprendizaje invisible que "busca dar mayor relevancia y visibilidad a ciertas prácticas de adquisición de saberes y a ciertas habilidades no institucionalizadas e incluso no necesariamente conscientes" (Cobo, 2011; p. 133); además de que constituye un proceso de innovación que puede ser un punto de partida para otras innovaciones y para aprovechar su potencial para la educación formal. Lo que reafirma lo relativo a una movilización del conocimiento en espacios distintos, nuevas ecologías favorecedoras del aprendizaje, con procesos formales, informales y no formales (Williams, Karousou \& Mackness, 2011; Sangrà \& Wheeler, 2013).
Sobre el alcance y la implicación organizacional los informantes dieron una valoración positiva de la gestión del foro en un espacio virtual que facilita el acceso, sin inversiones económicas para trasladarse y permanecer en lugares de realización, además de la flexibilidad del manejo del tiempo para ver las ponencias, al ser publicadas con anterioridad. Sin embargo, en lo concerniente a la interrogante sobre los aspectos que pudieran mejorarse en cuanto a la administración de las distintas fases del foro, en lo que respecta al tiempo se insistió en la necesidad de optimizarlo para ver y opinar sobre las ponencias; sobre esto, Crespo (2015) aludió "que con el fin de posibilitar un mayor número de consultas y debates, suelen ser prolongados en el tiempo...de esta forma se facilita que los participantes entren de vez en cuando, compatibilizándolo con sus actividades laborales" (p. 4).

Hubo diferentes percepciones en cuanto al manejo del tiempo, bien sea por su efecto economizador, por la posibilidad de alternar el foro con otras actividades cotidianas y la oportunidad de lectura previa de las ponencias, o que el tiempo previo para leer y opinar no fue suficiente, además de que los horarios para la actividad sincrónica debían consensuarse, así como exigir el cumplimiento del tiempo de intervención de ponentes; estas pueden ser algunas ideas congruentes con lo expresado por Godino \& Contreras (2017) acerca de las ventajas de la modalidad asincrónica, al permitir tiempo para elaborar las respuestas que otros participantes formulan a los trabajos, así como para la oportunidad de intercambio con los autores. Las otras percepciones traen a colación el manejo personal que se da al uso del tiempo, condición que debe ser autorregulada por el participante, sobre todo al desenvolverse en espacios virtuales; no obstante, hace pensar en la flexibilidad que debe darse al fijar cronogramas y crear alertas como recordatorios para ayudar a quienes se les dificulta la autogestión del tiempo.

En cuanto a las fases de planificación y de comunicación asincrónica y sincrónica, los hallazgos puntualizan la necesidad de revisar, ajustar y optimizar el foro invertido, en términos de su organización, estructura y funcionamiento. También se reconocieron las implicaciones negativas asociadas con la carencia de estrategias de difusión del foro para incrementar el número de participantes, además de estrategias incentivadoras 
que promuevan una mayor actividad de participación y proactividad. Sobre esto último, dice Crespo (2015): "efectivamente, lo más importante en un evento virtual es que la interacción entre los diferentes actores sea dinámica, intercambiando sin retraimiento comentarios, opiniones y sugerencias relevantes sobre las aportaciones hechas en las ponencias" (p. 4).

A modo de conclusión, podría apuntarse que el foro invertido de InterConectados, en su alcance social y educativo, triangula con el contenido y contexto (Cobo, 2016) elementos que avalan su proyección como herramienta de gestión y movilización de conocimiento, del cual pueden aprovecharse aquellas instancias cuyo objetivo apunte al reconocimiento y a la solución de problemas que afecten la calidad de vida de la sociedad en general o de comunidades particulares.

En esa dirección, InterConectados seguirá generando el foro con una evaluación permanente, además de atender la necesidad de mejorar los aspectos organizacionales, asociados con su estructura y funcionamiento. Su denominación como foro invertido trasciende al foro en su modalidad asíncrona, al añadirle el modo síncrono y, aunque en la literatura no se encuentre tal denominación, fue la asumida para distinguirlo como un evento académico virtual, donde los interesados pueden participar mediante dos fases -asincrónica y sincrónica- para discutir sobre una temática de interés asociada con investigaciones realizadas.

\section{Referencias}

Abeysekera, L. \& Dawson, P. (2015). Motivation and cognitive load in the flipped classroom: definition, rationale and a call for research. Higher Education Research \& Development, 34(1), 1-14. https://bit.ly/2va23uN

Adell, J. y Castañeda, L.(2012). Tecnologías emergentes, ¿pedagogías emergentes? En Hernández, J., Pennesi, M., Sobrino, D. y Vázquez, A. (Coord.), Tendencias emergentes en educación con TIC. (pp. 13-32). Barcelona: Asociación Espiral, Educación y Tecnología. https://bit. ly/3bj1L5m
Biesta, G.(2010). Good Education in an Age of Measurement: Ethics, Politics, Democracy. London: Routledge. Revista Interuniversitaria de Formación del Profesorado, 91(32), 114. https://bit.ly/ 2VTruNw

Anderson, C. R. \& McLachlan, S. M. (2016). Transformative research as knowledge mobilization: Transmedia, bridges, and layers. Action Research, 14(3), 295-317. Doi: https://doi.org/ $10.1177 / 1476750315616684$

Baker, J. W. (April, 2000). The 'classroom flip': Using web course management tools to become the guide by the side. 11 th International Conference on College Teaching and Learning, Jacksonville, Florida, United States. https://bit. ly/2OvWU6W

Bañuelos, A. \& Barron, H. (2005). Modelos de gestión del conocimiento para la educación en línea. Apertura, 4-54. https://bit.ly/30EhW7K

Brito, V. (2004). El foro electrónico: una herramienta tecnológica para facilitar el aprendizaje colaborativo. Edutec. Revista electrónica de tecnología educativa, (17), a038-a038. Doi: https://doi.org/ 10.21556/edutec.2004.17.532

Buil, I. Hernández, B., Sesé, F. J. \& Urquizu, P. (2012). Los foros de discusión y sus beneficios en la docencia virtual: recomendaciones para un uso eficiente. Innovar, 22(43), 131-143. https://bit. ly/2HEVsuE

Butt, A. (2014). Student views on the use of a flipped classroom approach: Evidence from Australia. Business Education \& Accreditation, 6(1), 33-43. https://bit.ly/2vc7GIE

Coll, C. (2013). La educación formal en la nueva ecología del aprendizaje: tendencias, retos y agenda de investigación. En Rodríguez Illera, J.L. (Comp.). Aprendizaje y educación en la sociedad digital. (pp. 156-170). Barcelona: Universitat de Barcelona. Doi: https://doi.org/ 10.1344/ 106.000002060

Cobo, C. (2011). Casos y experiencias para aprender. En Cobo, C. \& Moravec, J. (Comps). Aprendizaje Invisible: una nueva ecología de aprendizaje (pp. 107-140). Barcelona: Col.lecció Transmedia XXI. Laboratori de Mitjans Interactius /Publica- 
cions i Edicions de la Universitat de Barcelona. https://bit.ly/1qTVLVK

Cobo, C. (2016). La Innovación Pendiente. Reflexiones (y Provocaciones) sobre educación, tecnología y conocimiento. Montevideo: Colección Fundación Ceibal/Debate. https://bit.ly/2JZjNyj

Crespo, J. (2015). Eventos virtuales. Ideas alrededor del estado de la cuestión. ASRI. Arte y sociedad. Revista de investigación, 8(15), s/n. https://bit.ly/ 30Duv3m.

Díez, E. \& Ordońez, L. (2018). Las TIC como elemento motivador para el trabajo de las universidades con las comunidades: el caso de los foros invertidos de InterConectados. Ciencia y Educación, 2(1), 37-50. Doi: http://dx.doi.org/10.22206/ cyed.2018.v2i1.pp37-50

Dougherty, D. \& Dunne, D. D. (2011). Organizing ecologies of complex innovation. Organization Science, 22(5), 1214-1223. https://bit.ly/ 2HMUdtG

Drucker, P. F. (2000). Managing knowledge means managing oneself. Leader to leader, 16(2), 8-10.

Fernández, E. \& Martínez, J. (2018). Abriendo la investigación educativa a la pluralidad de contextos, agentes, y conocimientos. En Martínez, J. \& Fernández, E. (Comps). Ecologias de aprendizaje: educación expandida en contextos múltiples (pp. 9-26). https://bit.ly/2JxwCAs

Fisher, C. (2012). Knowledge brokering and intermediary concepts: analysis of an e-discussion on the Knowledge Brokers' Forum. Institute for Development Studies. Retrieved October 16, 2012. https://bit.ly/2wg7OnL

García, J. (2010). Gestión de la información y el conocimiento. Observatorio para la educación en ambientes virtuales. México. Universidad de Guadalajara Sistema de Universidad Virtual. https://bit.ly/30EZV9J.

Godino, J. D. \& Contreras, J. M. (2017). Uso de recursos virtuales en la difusión y construcción de conocimiento científico: el caso del Congreso CIVEOS. En Muñoz-Escolano, J. M., Arnal-Bailera, A., Beltrán-Pellicer, P., Callejo, M. L. \& Carrillo, J. (Eds.), Investigación en Educación Matemática XXI (p. 523). Zaragoza: SEIEM. https://bit.ly/2YFtYvV
Grant, R. M. (1996). Toward a knowledge-based theory of the firm. Strategic management journal, 17(S2), 109-122. https://bit.ly/2FL8atl.

Grant, R. M. (1996b). Prospering in dynamically-competitive environments: Organizational capability as knowledge integration. Organization science, 7(4), 375-387. https://bit.ly/ 2wej1Fy

Hayek, F. A. (1945). The use of knowledge in society. The American economic review, 35(4), 519-530. https://bit.ly/31GZEE9

Hernández, R., Fernández, C. \& Baptista, P. (2014). Metodología de la investigación. Ciudad de México: Mc Graw Hill.

Herreid, C. F. \& Schiller, N. A. (2013). Case studies and the flipped classroom. Journal of College Science Teaching, 42(5), 62-66. https://bit.ly/ $2 \mathrm{OyUdBx}$

Karabulut-Ilgu, A., Jaramillo Cherrez, N. \& Jahren, C. T. (2018). A systematic review of research on the flipped learning method in engineering education. British Journal of Educational Technology, 49(3), 398-411. https://bit.ly/39efokg

Martínez, W., Esquivel-Gámez, I. \& Martínez-Castillo, J. (2014). Aula invertida o modelo invertido de aprendizaje: origen, sustento e implicaciones. Los Modelos Tecno-Educativos, revolucionando el aprendizaje del siglo XXI, 143-160. https://go. aws/2S4AXOI

Milman, N. B. (2012). The flipped classroom strategy: What is it and how can it best be used? Distance learning, 9(3), 85. https://go. aws/2S4uAek

Nonaka, I. \& Takeuchi, H. (1995). The Knowledge-creating company: how Japanese companies create the dynamics of innovation, New York: Oxford University Press. https://bit. ly/2HuYClX

O'Flaherty, J. \& Phillips, C. (2015). The use of flipped classrooms in higher education: A scoping review. The internet and higher education, 25, 85-95. https://bit.ly/2UxI2c6

Ordóñez, L. A. (2016). Colaboración y TIC en América Latina: el caso Interconectados. Revista Linhas, 17(33), 82-110. https://bit.ly/2JxQ9kf 
Padrón, J. (2016). (Presentación web) Para una teoría de investigación: Modelo de Variabilidad de la Investigación Cientifica. https://bit.ly/2wcE6QH

Quirós, E. (2009). Recursos didácticos digitales: medios innovadores para el trabajo colaborativo en línea. Revista Electrónica Educare, 13(2), 47-62. https://bit.ly/2ErY1PY.

Rodríguez, M., García, F., Pérez, M. \& Castillo, J. (2009). La gestión del conocimiento factor estratégico para el desarrollo. Gestión en el tercer milenio, 12(23), 7-14. https://bit.ly/2M1YqPn

Sangrà, A. \& Wheeler, S. (2013). New Informal Ways of Learning: Or Are We Formalizing the Informal? International Journal of Educational Technology in Higher Education, 10(1), 286-293. https://bit.ly/2YFkvVq

Shaxson, L., Bielak, A., Ahmed, I., Brien, D., Conant, B., Fisher, C. \& Phipps, D. (April, 2012). Expanding our understanding of $\mathrm{K}^{*}(\mathrm{Kt}, \mathrm{KE}$, $\mathrm{Ktt}, \mathrm{KMb}, \mathrm{KB}, \mathrm{KM}$, etc.). En A concept paper emerging from the $\mathrm{K}^{*}$ conferencia llevada a cabo en UNU-INWEH, Hamilton, ON.

Tójar, J. (2006). Investigación cualitativa: comprender y actuar. Madrid: Editorial La Muralla, S. A.

Williams, R., Karousou, R. \& Mackness, J. (2011). Emergent learning and learning ecologies in Web 2.0. The International Review of Research in Open and Distributed Learning, 12(3), 39-59. Doi: https://doi.org/10.19173/irrodl. v12i3.883

Wu, D. \& Hiltz, S. R. (2004). Predicting learning from asynchronous online discussions. Journal of Asynchronous Learning Networks, 8(2), 139-152. https://bit.ly/2JBPUoi.

Zainuddin, Z. \& Halili, S. H. (2016). Flipped classroom research and trends from different fields of study. International Review of Research in Open and Distributed Learning, 17(3), 313-340. https://bit.ly/39f0vOQ 